PO-0645 COMPLICATIONS OF PROLONGED PREGNANCY ON THE GROWTH RESTRICTED FETUS

${ }^{1} \mathrm{~L}$ Radulescu, ${ }^{2} \mathrm{O}$ Munteanu, ${ }^{3} \mathrm{~F}$ Popa, ${ }^{2} \mathrm{M}$ Cirstoiu. ${ }^{1}$ Biochemistry, UMF Carol Davila, Bucharest, Romania; ${ }^{2}$ Department of Obstetrics and Gynecology, Bucharest Emergency University Hospital, Bucharest, Romania; ${ }^{3}$ Department of Surgery, St. Pantelimon Clinical Emergency Hospital, Bucharest, Romania

10.1136/archdischild-2014-307384.1286

Backround Prolonged pregnancy is defined as any pregnancy exceeding 294 days or more. It is now well known that prolonged pregnancy is associated with an increased risk of perinatal mortality and morbidity, both maternal and fetal, particularly in intrauterine growth restricted fetuses.

Material and methods We analysed in a retrospective study all births complicated by intrauterine growth restriction of all births in the period 2010-2012. Criteria for inclusion in the study were represented by the diagnosis of intrauterine growth restriction and prolonged pregnancy.

Results Rates of transient tachypnea, hypoxia, neonatal apnea, sepsis, thrombocytopenia and hypoglycemia were higher in the postdated growth restricted newborns. An increased frequency of children who needed intensive care after birth, a longer period of hospitalisation and a higher frequency of oligohydramnios were observed in patients who associated both pathologies. Conclusions Chronologically prolonged pregnancies associated with intrauterine growth restriction represent a pathology that requires close monitoring in order to anticipate possible materno-fetal complications.

\section{PO-0646 ACOUSTIC ENVIRONMENT STUDY IN A NEONATAL INTENSIVE CARE UNIT}

${ }^{1} \mathrm{~N}$ Torre, ${ }^{1} \mathrm{~A}$ Riverola, ${ }^{2} \mathrm{G}$ Raboshchuk, ${ }^{1} \mathrm{~B}$ Muñoz, ${ }^{2} \mathrm{C}$ Nadeu, ${ }^{3} \mathrm{~S}$ Navarro. ${ }^{1}$ Neonatology, Hospital Sant Joan de Deu, Barcelona, Spain; ${ }^{2}$ TALP Research Center Department of Signal Theory and Communications, Universitat Politecnica de Catalunya, Barcelona, Spain; ${ }^{3}$ Departament de Enginyeria, Hospital Sant Joan de Deu, Barcelona, Spain

10.1136/archdischild-2014-307384.1287

Background and aims NICU's environment is rich from an acoustical point of view. Long-termeffects of exposure to repeated noise in preterm's neurological and sensorial developmentis of concern.

We aimed to analyse the acoustic environment of the NICU, the types ofsounds present in it and their characteristics (both intensity and frequency).

Patients and methods Ten recording sessions were carried out. Duration of acquired data:108.7 min. Two microphones connected to the Olympus LS-5 Linear-PCM Recorder were used. One microphone was placed inside the incubator; the other, outside.

Results More than 65 different sounds were found. We divided them intoacoustically homogeneous groups:

1. Tones (mostly equipment alarms), were present in $21 \%$ of the totalrecorded time.

2. Vocalisations (with a lot ofdifferent subclasses here: speech, cry, laugh, cough... both from infants and adults) in 63\%.

3. Other (all the other sounds: equipment moving, respiratory devices, caregiving tasks, etc.) in $70 \%$.

Average/Maximum sound intensity was $54 / 72.3 \mathrm{~dB}$ inside the incubator and 51.6/69.7 $\mathrm{dB}$ outside.
With respect to the spectral domain, the fundamental frequencies of alarmsare mostly around $1.5 \mathrm{kHz}$, and the content of vocalisations can be observed upto $8 \mathrm{kHz}$. The group other is diverse, since it contains lower- andhigher-frequency sounds, and also sounds spread over awide frequency range (e.g. ventilation noise).

Conclusions 1. In our NICU noise levels exceeds recommendations.

2. There is a large and diverse set of sounds; vocalisations are the most common.

3. Noise inside the incubator is higher than outside it.

\section{PO-0647 TO DETERMINE THE INCIDENCE, RISK FACTORS AND NEED FOR SURGERY FOR RETINOPATHY OF PREMATURITY(ROP) AMONG VERY-LOW-BIRTH-WEIGHT (VLBW) INFANTS WEIGHING $<1500 \mathrm{GMS}$}

${ }^{1} \mathrm{~V}$ Shah, ${ }^{2} \mathrm{~J}$ Chiang, ${ }^{3} \mathrm{~S}$ Chung, ${ }^{4} \mathrm{Y}$ Ling. ${ }^{1}$ Neonatal and Dev Medicine, Singapore General Hospital, Singapore, Singapore; ${ }^{2}$ Neonatal and Dev Medicine, Yong Loo Lin Medical School, Singapore, Singapore; ${ }^{3}$ Health Services Research and Biostatistics Unit Division of Research, Singapore General Hospital, Singapore, Singapore; ${ }^{4}$ Adult Strabismus and Paediatric Ophthalmology, Singapore National Eye Center, Singapore, Singapore

\subsection{6/archdischild-2014-307384.1288}

Materials and methods Retrospective analysis of 12 years data, of all VLBW screened for ROP, managed by Singapore General Hospital, for maternal, neonatal risk factors using univariate and multiple logistic regressions.

Results Incidence of ROP was $24.8 \%$ among all screened VLBW. By univariate analysis, maternal risk factors for severe ROP were prolonged rupture of membrane (PROM), pyrexia, multiple births, tocolysis, fetal disress and vaginal delivery. Neonatal risk factors for ROP were, infant with lower gestational age than 25 \pm 2 weeks (mean $+\mathrm{SD}$ ), birth weight lesser than $689 \pm 147$ gm (mean + SD), low Apgar scores, hyaline membrane disease (HMD) requiring surfactant, hypothermia, sepsis, hypotension, patent ductus arteriosus (PDA), air-leak, hypoglycaemia, intraventricular haemorrhage (IVH) increased days on assisted ventilation, CPAP, Oxygen and high mean $\mathrm{FiO}_{2}$ and Chronic lung disease (CLD), are significant risk factors for ROP $(\mathrm{p}<0.05)$.

By multivariate analysis, lower GA $(\mathrm{OR}=0.728,95 \%$ $\mathrm{CI}=0.609-0.870)$, lower $\mathrm{BW}(\mathrm{OR}=0.996,95 \% \mathrm{CI}=0.994$ 0.997 ) and increased days on oxygen (OR $=1.015,95 \%$ $\mathrm{CI}=1.006-1.025)$ were independent risk factor for ROP.

Conclusion High risk factors for severe ROP are extreme preterm infants born vaginally to mother with infection and PROM, multiple birth, used tocolysis, fetal distress with lower GA than $25+2$ weeks (mean+SD), with BW lesser than 689 +147 gm mean+SD), having low 1,5 min Apgar score, HMD requiring surfactant, hypothermia, sepsis, hypotension, patent ductus arteriosus (PDA), chronic lung disease (CLD), air-leak, hypoglycaemia, intraventricular haemorrhage (IVH). Longer duration of mechanical ventilation, CPAP and oxygen with increased FIO2 due to CLD, are also higher risk of ROP. By multivariate analysis lower GA, BW and increased days on oxygen were found to be high risk factors for ROP. Prevention of extreme prematurity, maternal infection, PROM, optimal ventilator care, with careful titration of oxygen therapy which can decrease CLD may reduce the incidence and severity of ROP in these high-risk ELBW infants. 